OPEN ACCESS

Edited by:

Dominique Debanne, INSERM U1072 Neurobiologie des Canaux loniques et de la Synapse, France

Reviewed by:

Matthew N. Rasband, Baylor College of Medicine,

United States

Juan José Garrido,

Spanish National Research Council

(CSIC), Spain

Hiroshi Kuba,

Nagoya University, Japan

${ }^{*}$ Correspondence:

Catherine Faivre-Sarrailh

catherine.sarrailh@univ-amu.fr

tPresent address:

Giulia Bonetto,

Wellcome - MRC Cambridge Stem Cell Institute, University of Cambridge,

Cambridge, United Kingdom Bruno Hivert,

CNRS UMR7289, Institut

de Neurosciences de la Timone, Aix-Marseille Université, Marseille,

France

Specialty section:

This article was submitted to Cellular Neurophysiology,

a section of the journal

Frontiers in Cellular Neuroscience

Received: 22 February 2019 Accepted: 02 May 2019

Published: 16 May 2019

Citation:

Bonetto G, Hivert $B$

Goutebroze L, Karagogeos D, Crépel V and Faivre-Sarrailh C (2019) Selective Axonal Expression of the Kv1 Channel Complex in Pre-myelinated GABAergic Hippocampal Neurons.

Front. Cell. Neurosci. 13:222. doi: 10.3389/fncel.2019.00222

\section{Selective Axonal Expression of the Kv1 Channel Complex in Pre-myelinated GABAergic Hippocampal Neurons}

\author{
Giulia Bonetto ${ }^{1 t}$, Bruno Hivert ${ }^{1 t}$, Laurence Goutebroze ${ }^{2}$, Domna Karagogeos ${ }^{3}$, \\ Valérie Crépel ${ }^{1}$ and Catherine Faivre-Sarrailh ${ }^{1 *}$ \\ ${ }^{1}$ INSERM UMR1249, Institut de Neurobiologie de la Méditerranée, Aix-Marseille Université, Marseille, France, ${ }^{2}$ INSERM \\ UMR-S 1270, Institut du Fer à Moulin, Faculté des Sciences et Ingénierie, Sorbonne Université, Paris, France, ${ }^{3}$ Department \\ of Basic Sciences, Institute of Molecular Biology and Biotechnology, Foundation for Research and Technology, University \\ of Crete Medical School - University of Crete, Heraklion, Greece
}

In myelinated fibers, the voltage-gated sodium channels Nav1 are concentrated at the nodal gap to ensure the saltatory propagation of action potentials. The voltage-gated potassium channels Kv1 are segregated at the juxtaparanodes under the compact myelin sheath and may stabilize axonal conduction. It has been recently reported that hippocampal GABAergic neurons display high density of Nav1 channels remarkably in clusters along the axon before myelination (Freeman et al., 2015). In inhibitory neurons, the Nav1 channels are trapped by the ankyrinG scaffold at the axon initial segment (AIS) as observed in pyramidal and granule neurons, but are also forming "pre-nodes," which may accelerate conduction velocity in pre-myelinated axons. However, the distribution of the Kv1 channels along the pre-myelinated inhibitory axons is still unknown. In the present study, we show that two subtypes of hippocampal GABAergic neurons, namely the somatostatin and parvalbumin positive cells, display a selective high expression of Kv1 channels at the AIS and all along the unmyelinated axons. These inhibitory axons are also highly enriched in molecules belonging to the juxtaparanodal Kv1 complex, including the cell adhesion molecules (CAMs) TAG-1, Caspr2, and ADAM22 and the scaffolding protein 4.1B. Here, taking advantage of hippocampal cultures from 4.1B and TAG-1 knock-out mice, we observed that 4.1B is required for the proper positioning of Caspr2 and TAG-1 along the distal axon, and that TAG-1 deficiency induces alterations in the axonal distribution of Caspr2. However, the axonal expression of Kv1 channels and clustering of ankyrinG were not modified. In conclusion, this study allowed the analysis of the hierarchy between channels, CAMs and scaffolding proteins for their expression along hippocampal inhibitory axons before myelination. The early steps of channel compartmentalization preceding myelination may be crucial for stabilizing nerve impulses switching from a continuous to saltatory conduction during network development.

Keywords: Caspr2, TAG-1, protein 4.1B, juxtaparanodes, parvalbumin, somatostatin, interneuron 


\section{INTRODUCTION}

In myelinated fibers, ion channels are targeted to precise sub-cellular compartments at the axon initial segment (AIS) and nodes of Ranvier contributing to safe action potential propagation. At the node of Ranvier, the voltage-gated $\mathrm{Na}^{+}$ (Nav) channels are enriched at the nodal gap to ensure saltatory conduction, while the $\mathrm{K}^{+}$channels are localized at nodes and juxtaparanodes to secure spike propagation (Rasband, 1998; Devaux and Gow, 2008). On both sides of the node, the paranodal junctions restrict the lateral diffusion of $\mathrm{Na}^{+}$ and $\mathrm{K}^{+}$channels and preclude current leakage across the paranodes (Salzer, 2008). The segregation of ion channels at the nodes of Ranvier is induced by contacts with the myelinating glial cells (Ching et al., 1999). Moreover, the localization of the $\mathrm{Na}^{+}$and $\mathrm{K}^{+}$channels is strongly dependent on cell adhesion molecules (CAMs) at nodes, paranodes, and juxtaparanodal regions of myelinated axons (Eshed-Eisenbach and Peles, 2013). Specifically, the nodal concentration of $\mathrm{Na}^{+}$ channels depends on the interactions between the axonal CAM neurofascin 186 and extracellular matrix proteins (Sherman et al., 2005; Feinberg et al., 2010). Neurofascin 186 clustering recruits the scaffolding proteins ankyrinG and BIV spectrin which in turn mediate the sequestration of Nav channels. In addition, the lateral diffusion of nodal $\mathrm{Na}^{+}$channels is restricted by the axo-glial junctions at paranodes, which are formed by the axonal proteins Caspr/contactin and the glial neurofascin 155 (Bhat et al., 2001; Charles et al., 2002).

The trapping of voltage-gated $\mathrm{K}^{+}(\mathrm{Kv}) \mathrm{Kv} 1.1 / \mathrm{Kv} 1.2$ channels at the juxtaparanodal regions depends on axo-glial interactions mediated by CAMs including TAG-1/contactin2/CNTN2 and Caspr2/CNTNAP2 (Poliak et al., 2001, 2003; Traka et al., 2003; Horresh et al., 2008). Within the axonal membrane, TAG-1 forms a cis-complex with Caspr2, which allows the arrangement of a ternary complex with the glial-secreted form of TAG-1 (Tzimourakas et al., 2007; Savvaki et al., 2010). Disruption of either Caspr2 or TAG-1 in knock-out (KO) mice prevents the proper clustering of Kv1 channels at juxtaparanodes (Poliak et al., 2003; Traka et al., 2003). Moreover, TAG-1-deficient animals show alteration of myelinated axon conduction in the corpus callosum (Zoupi et al., 2018), as well as behavioral deficits and defects in gating and motor coordination (Savvaki et al., 2008). Similarly, the loss of Caspr2 is associated with defects in the propagation of action potentials along myelinated axons in the corpus callosum, with a slow-down of the repolarisation phase (Scott et al., 2017).

A number of scaffolding proteins, including band 4.1B, $\alpha \mathrm{II}$, and BII spectrin are expressed at paranodes and juxtaparanodes (Denisenko-Nehrbass et al., 2003; Ogawa et al., 2006; Zhang et al., 2013). In 4.1B-null mice the accumulation of TAG-1, Caspr2, and $\mathrm{Kv} 1$ at juxtaparanodes is altered, indicating the crucial role of this protein in the formation of the juxtaparanodal domain (Horresh et al., 2010; Buttermore et al., 2011; Cifuentes-Diaz et al., 2011; Einheber et al., 2013). A dynamic and precise subcompartmentalization of Kv1 channels that may help to regulate the conduction occurs in developing myelinated axons (Vabnick et al., 1999). Precisely, Kv1 channels and Caspr2 are first enriched at paranodes and progressively restricted to juxtaparanodes, while they could be seen transiently trapped between heminodes and at the newly formed nodal zone. In contrast, the distribution of the scaffolding protein $4.1 \mathrm{~B}$ is restricted to internodes and preferentially co-localized with Caspr at paranodes (Hivert et al., 2016). The transient localization of Kv1 channels at nodes and paranodes may be directly involved in speeding repolarisation to allow trains of action potentials (Vabnick et al., 1999).

In inhibitory neurons, the myelination processes as well as the mechanisms leading to the segregation of ion channels at the nodes of Ranvier have been poorly investigated. It has been recently reported that pre-myelinated hippocampal GABAergic neurons, including parvalbumin (PV) and somatostatin (SST) cells, display high density of Nav1 channels associated with ankyrinG in clusters (the so-called "pre-nodes") along the axon (Freeman et al., 2015). Adhesive contact with ensheathing myelinating cells is a prerequisite for the nodal trapping of $\mathrm{Na}^{+}$ channels in pyramidal neurons whereas pre-nodal clusters can be selectively induced in GABAergic neurons by oligodendroglial secreted factors. Interestingly, the presence of Nav1 clusters is correlated with an acceleration of conduction in pre-myelinated inhibitory axons (Freeman et al., 2015).

In the present study, we examine the distribution of the Kv1 complex in hippocampal pre-myelinated inhibitory neurons. We show that GABAergic neurons, namely the PV and SST cells, selectively display high concentration of Kv1 channels all along their axon and the associated molecules, TAG-1, Caspr2, ADAM22, and protein 4.1B. Furthermore, we demonstrate that in these pre-myelinated inhibitory neurons, TAG-1 is required for the proper distribution of Caspr2, while 4.1B is necessary for the correct localization of both Caspr2 and TAG-1. Interestingly, we found that TAG-1 expression in vivo is constrained to specific CA1 hippocampal layers; it is selective to the SST cells in the stratum oriens and the PV cells in the stratum pyramidale and may be possibly related to a pre-myelinated phenotype. The specific expression of CAMs associated with the Kv1 channels in the GABAergic neurons may help to secure conduction during network development.

\section{MATERIALS AND METHODS}

\section{Animals}

The care and use of rats and mice in all experiments were carried out according to the European and Institutional guidelines for the care and use of laboratory animals and approved by the local authority (laboratory's agreement number D13-055-8, Préfecture des Bouches du Rhône). The following rat and mouse strains were used in this study: Wistar rats and C57bl/6 mice (Janvier Breeding Center), Tag-1 KO mice (Traka et al., 2003), and 4.1B KO mice (Cifuentes-Diaz et al., 2011).

\section{Cell Culture}

Primary mixed hippocampal cell cultures were prepared from embryonic day 18 Wistar rats. Hippocampi were collected in Hanks' balanced salt solution, dissociated with trypsin and plated at a density of $1.210^{5}$ cells $/ \mathrm{cm}^{2}$ on poly-L-lysine (Sigma-Aldrich, 
Merck) coated coverslips. Hippocampal neurons were cultured in Neurobasal supplemented with $2 \%$ B-27, $1 \%$ penicillinstreptomycin and $0.3 \%$ glutamine in a humidified atmosphere containing $5 \% \mathrm{CO} 2$ at $37^{\circ} \mathrm{C}$. The mixed hippocampal cultures were maintained for 3-4 weeks in vitro and contained astrocytes and oligodendrocytes. Unless specified, all culture reagents were purchased from Gibco, Thermo Fisher Scientific. Once a week half of the culture medium was replenished. Hippocampal cell cultures were prepared from embryonic day 16 wild type, Tag$1 \mathrm{KO}$ (Traka et al., 2003) or 4.1B KO mice (Cifuentes-Diaz et al., 2011) using the same protocol. At least, three different hippocampal cell cultures were performed from wild type and KO mice and processed in parallel on the same days for immunofluorescence staining.

\section{Antibodies and Immunofluorescence Staining}

The following primary antibodies were used: rabbit antiserum against protein 4.1B (Cifuentes-Diaz et al., 2011), rabbit antiTAG-1 TG3 (Buttiglione et al., 1998), and rabbit anti-ankyrinG, a gift from Dr. Gisèle Alcaraz. Human anti-Caspr2 antiserum was previously described (Pinatel et al., 2015). Mouse antipanNav mAb (clone K58/35) was purchased from Sigma, chicken anti-MAP2 antibody (ab5392) and rat anti-MBP mAb (ab7349) from Abcam, rabbit anti-Prox1 antibody (ab5475) from Millipore, goat anti-PV antibody (PVG-214) from Swant, goat anti-SST mAb (sc55565) from Santa-Cruz. Mouse anti-Kv1.1 (clone K20/78), anti-Kv1.2 (clone K14/16), anti-Kv1.4 (clone $\mathrm{K} 13 / 31$ ), anti-ADAM22 (clone N46/30), and anti-ankyrinG (clone N106/36) mAbs were obtained from NeuroMab (UC Davis/NIH NeuroMab Facility). Mouse anti-TAG-1 (1C12) and anti-GAD65 (GAD6) mAbs were from Developmental Studies Hybridoma Bank. AlexaFluor-405, -488, -568 and 647-conjugated secondary antibodies were purchased from Molecular Probes.

Live immunostaining was performed using mouse antiTAG-1 1C12 (1:2000), rabbit anti-TAG-1 TG3 (1:400), or human anti-Caspr2 (1:400) antibodies for $30 \mathrm{~min}$ and with secondary antibodies (1:800) for $30 \mathrm{~min}$ diluted in culture medium at room temperature. Cells were fixed with 1 or $4 \%$ paraformaldehyde in PBS for $10 \mathrm{~min}$ and permeabilized with $0.1 \%$ Triton-X100 for $10 \mathrm{~min}$. Immunofluorescence staining on fixed neurons was performed using rabbit anti-ankyrinG (1:400), rabbit anti-4.1B (1:2000), rabbit anti-prox1 (1:2000), chicken anti-MAP2 (1:10,000) antibodies, rat anti-MBP mAb (1:200), mouse anti-panNav (1:500), anti-Kv1.1, anti-Kv1.2, anti-Kv1.4, anti-ADAM22, anti-ankyring (1:100) mAbs for $60 \mathrm{~min}$ and with AlexaFluor-conjugated secondary antibodies for $30 \mathrm{~min}$ diluted in PBS with $3 \%$ bovin serum albumin. Immunostaining with goat anti-PV (1:500) or mouse anti-SST (1:200) antibodies was performed overnight at $4^{\circ} \mathrm{C}$.

\section{In vivo Study and Immunohistochemistry}

For the in vivo study, P21 $(n=3)$ rats were deeply anesthetized with a mix of ketamine-xylazine (Vetoquinol) and then transcardially perfused with PBS followed by $4 \%$ paraformaldehyde in PBS. Brains were removed and placed in the same fixative for $30 \mathrm{~min}$, then cryoprotected by infiltration in $30 \%$ sucrose overnight, embedded in $7.5 \%$ gelatin- $15 \%$ sucrose, and immediately frozen in a dry ice-isopentane bath. Thirty micron-thick cryostat sections were mounted on SuperfrostVR Plus microscope slides (Thermo Fisher Scientific), permeabilized by immersion in ice-cold acetone at $-20^{\circ} \mathrm{C}$ for $10 \mathrm{~min}$, blocked for $1 \mathrm{~h}$ in $5 \%$ bovine serum albumin containing $0.5 \%$ Triton $\mathrm{X}$ 100 in PBS, and incubated overnight at $4^{\circ} \mathrm{C}$ with combinations of the following primary antibodies: goat anti-PV (1:500), goat anti-SST (1:500), mouse anti-TAG-1 (1C12, 1:2000), human antiCaspr2 (1:200), mouse anti-Kv1.2 (1:100), rabbit anti-ankyrinG (1:400), rat anti-MBP (1:200). Slides were then washed and incubated with the appropriate AlexaFluor-conjugated secondary antibodies (1:800) for $2 \mathrm{~h}$. Slides were covered with Vectashield mounting medium (Vector Laboratory), which contains DAPI to visualize cell nuclei.

\section{Image Acquisition and Statistical Analysis}

Image acquisition was performed on a Zeiss laser-scanning microscope LSM780 equipped with $63 \times 1.32$ NA oil-immersion objective for cell culture and $20 \times$ or $63 \times$ objectives for hippocampal slice imaging. Images of AlexaFluor-stained cells were obtained using the $488 \mathrm{~nm}$ band of an Argon laser and the 405, 568, and $647 \mathrm{~nm}$ bands of a solid-state laser for excitation. Fluorescence images were collected automatically with an average of two-frame scans at airy 1. Maximum intensity projection of images and plot profiles of immunofluorescence intensity (10 pixels width) were carried out using Image J software $(\mathrm{NIH})$. Images are single confocal sections unless the number of $\mathrm{z}$-steps is indicated.

The percentage of SST and PV neurons positive for TAG1, Caspr2, and Kv1.2 was determined by examining at least 50 neurons on 2 coverslips per condition (wild type, TAG-1 KO and $4.1 \mathrm{~B} \mathrm{KO}$ mice). 4-z step confocal sections (410 $\mathrm{nm}$ ) were acquired with the same settings (laser intensity and gain) and maximum intensity projections were generated before analysis. Results were expressed as mean \pm SEM of at least three independent experiments. Statistical analyses were performed using the GraphPad Prism software. The data normal distribution was tested using d'Agostino and Pearson's test. The Student's paired $t$-test or the one-way analysis of variance (ANOVA), followed by Dunnett post hoc test was performed.

\section{RESULTS}

\section{The Kv1.2 Subunits Are Highly Expressed Along Inhibitory Axons in Hippocampal Cultures}

We examined the distribution of the Kv1 channels in hippocampal cell culture at Day in vitro DIV21, a representative day before myelination onset. It has been reported that Kv1.2 is concentrated at the AIS in subpopulations of hippocampal neurons after DIV10 (Ogawa et al., 2008; 
Sanchez-Ponce et al., 2012). Here, we showed that Kv1.2 immunostaining was restricted at the AIS in some neurons (Figures 1A,B, yellow arrows). Kv1.2 was detected at the AIS of granule cells identified using immunostaining for the transcription factor proxl (Figure 1D, yellow arrow). Strikingly, the Kv1.2 channels were also strongly expressed along the axon in some neurons identified as GABAergic neurons using immunostaining for GAD65 (Figures 1A,C, red arrows). We showed that these neurons were either $\mathrm{PV}^{+}$ or $\mathrm{SST}^{+}$interneurons as illustrated for $\mathrm{PV}^{+}$neurons in Figure 1E. The inhibitory axons displayed distal clusters of Nav channels and ankyring (Figures 1F,G, red arrows) as it has been described specifically in pre-myelinated GABAergic neurons in hippocampal cultures (Freeman et al., 2015). We analyzed more precisely the distribution of Kv1.2 relatively to the ankyrinG clusters along the inhibitory axons at DIV21. As shown in Figure 1G, immunostaining for Kv1.2 after fixation and permeabilization indicated that these channels were enriched at the AIS co-localized with ankyrinG and homogenously distributed along the axon. Plot profile analysis illustrates that Kv1.2 was concentrated at the AIS together with ankyrinG and uniformly present along the axon irrespectively to peaks corresponding to ankyrinG clusters (Figure $\mathbf{1 H}$, red arrows).

We noticed that the GABAergic neurons showing Kv1.2 distribution all along their axon also exhibited high expression of the Kv1.2 channel at the level of their soma by comparison with excitatory neurons that displayed AIS-restricted expression of Kv1.2 (Figure 1A). We estimated the immunofluorescence intensity of Kv1.2 in the soma of GAD65-positive neurons $(n=30)$ relatively to those of other neurons in the same area $(n=93)$ and observed that it was increased by $33 \pm 4 \%$. These data suggest that the axonal distribution of Kv1.2 channels may be correlated with the level of expression in the cell body.

Kv1 channels exist as homomeric and heteromeric complexes in neurons and distinct Kv1 channels could be selectively addressed at the axonal membrane based on their subunit composition (Jenkins et al., 2011). In addition to Kv1.2, the Kv1.1, and Kv1.4 subunits have been reported to be concentrated at the AIS of cultured hippocampal neurons (Ogawa et al., 2008). Indeed, we observed that Kv1.1 was expressed at the AIS of excitatory neurons (Figure 2A, yellow arrows). However, Kv1.1 was weakly expressed at the AIS and along the axons of GAD65-positive inhibitory neurons by comparison with Kv1.2 (Figures 2A,B) whereas it was mainly detected as vesicles in the somato-dendritic compartment of these cells (Figure 2A). The Kv1.4 subunit was colocalized with ankyring at the AIS of excitatory neurons (Figures 2C,D, yellow arrows), but very faintly expressed in GAD65- or SST-positive neurons (Figures 2C,D, red arrows). Altogether, these results suggest that Kv1 channels expressed in inhibitory axons in hippocampal cell cultures are composed mainly of Kv1.2 subunits.

Next, we asked whether the high level of Kv1.2 along inhibitory axons may be correlated with myelination or occurred before myelination onset using immunostaining for MBP as a marker of myelinating oligodendrocytes (Figure 3A). Mixed hippocampal cultures were performed in standard conditions but with plating at high density. These cultures contained glial cells, including few oligodendrocytes identified using immunostaining for the myelin basic protein (MBP) (Figure 3, red). However, at DIV 21, only very few neurons presenting high level of Kv1.2 along their axon were myelinated and in this case Kv1.2 only formed clusters along the myelinated segments (Figure 3B, green arrows in inset).

\section{TAG-1, Caspr2, ADAM22, and Protein 4.1B Are Selectively Expressed Along Inhibitory Axons in Cultured Hippocampal Neurons}

We investigated whether the cell adhesion and scaffolding molecules associated with the Kv1 channels at the juxtaparanodes in myelinated fibers may be also selectively expressed by inhibitory neurons. We previously reported that Caspr2 is preferentially expressed by GABAergic neurons in hippocampal cell cultures. Using live immunostaining with anti-Caspr2 antibodies from patients affected by autoimmune encephalitis, we have shown that Caspr2 is detected all along the axolemma including at the presynaptic terminals of inhibitory neurons (Pinatel et al., 2015). Here, we investigated the cell-type specific expression of TAG-1, which interacts both in cis and in trans with Caspr2 and may be either cis-associated with Caspr2 along the inhibitory axons or trans-interacting at the postsynapse with presynaptic Caspr2 (Traka et al., 2003; Savvaki et al., 2010; Pinatel et al., 2015). Live immunostaining was performed using mouse anti-TAG-1 $1 \mathrm{C} 12 \mathrm{mAb}$ at DIV21. As observed for Kv1.2 channels, TAG-1 was strongly expressed along the axon of some neurons, which displayed ankyring at the AIS as well as organized in regularly spaced clusters along the entire axonal length (Figures $\mathbf{4 A , B}$, red arrowheads). Surface double-immunostaining was performed using anti-TAG-1 mAb and human anti-Caspr2 antibodies, and indicated that the two CAMs were co-expressed along the same axons (Figure 4B). We hypothesized that these axons may belong to inhibitory neurons since exhibiting axonal clusters of ankyring. This was confirmed using doubleimmunostaining for TAG-1 and GAD65 (Figure 4C). In addition, TAG-1 and Caspr 2 were co-localized at the inhibitory pre-synaptic terminals surrounding the soma of pyramidal cells (Supplementary Figure S1). Next, using immunostaining with antibodies directed against SST and PV, we determined that both subtypes of inhibitory cells displayed high level of expression of TAG-1 along their axons (Figures 4F,G). As observed for Kv1.2, we noticed that the axonal distribution of TAG-1 along inhibitory axons also occurred before myelination (Figure 3C).

In addition to be highly expressed along inhibitory axons, TAG-1 was also expressed in a subpopulation of neurons at the AIS (approximately 30\%) (Figures 4A,D, yellow arrows). In contrast to what has been observed for Kv1.2 (Figure 1D), the granule cells identified with the marker proxl were negative for TAG-1 (Figure 4E).

We then investigated more precisely the relative distribution of TAG-1 and Caspr2 along inhibitory axons at DIV21 and observed that the two CAMs were strongly co-localized (Figure 5B). However, only TAG-1 was enriched at the AIS as 

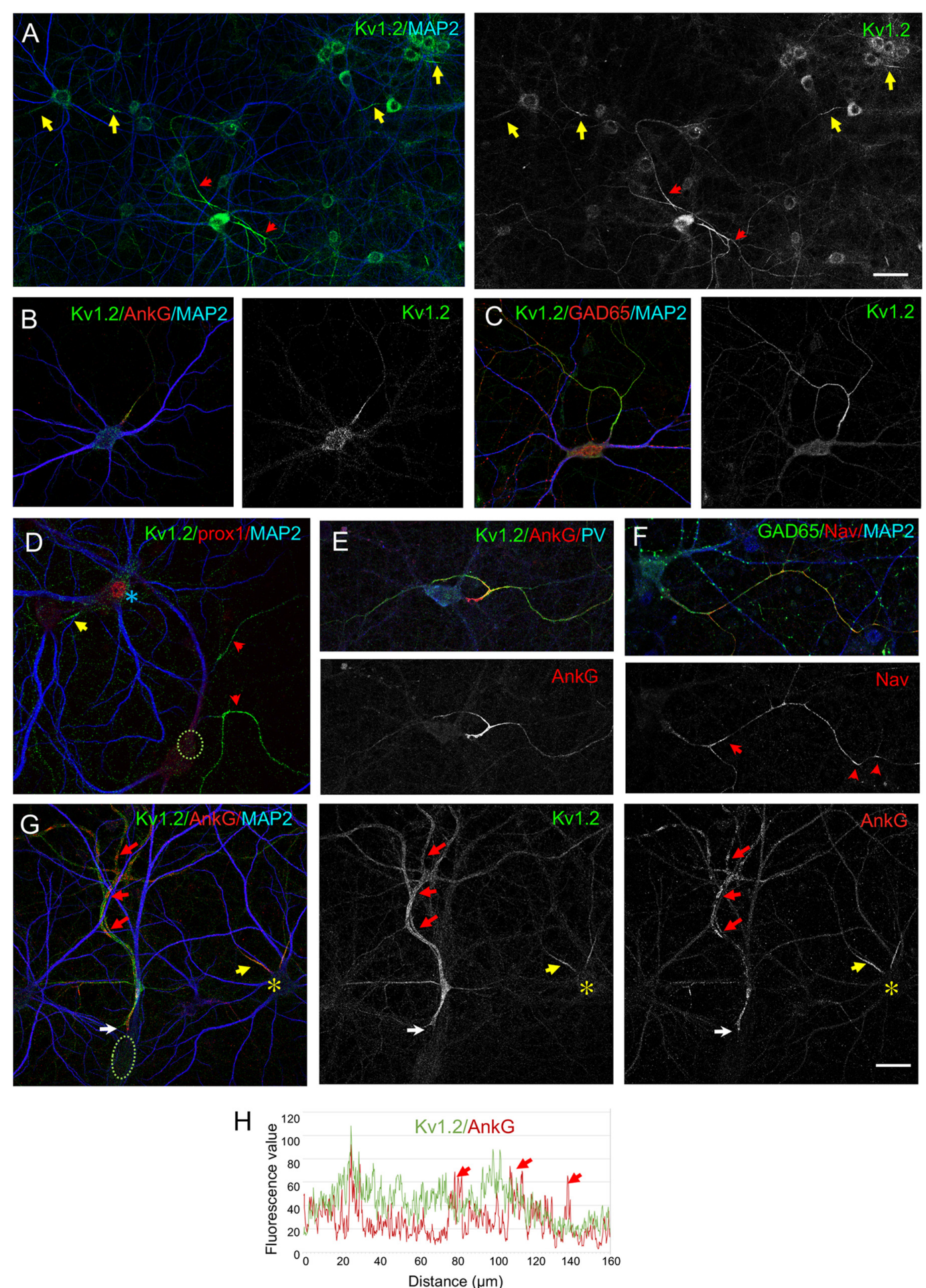

FIGURE 1 | Cell-type specific distribution of Kv1.2 in cultured hippocampal neurons. Hippocampal neurons at DIV21 were fixed, permeabilized and immunostained for Kv1.2 (green). MAP2 (blue) is used as a somato-dendritic marker and ankyrinG (AnkG) (B,E,G, red) as a marker of AlS. Kv1.2 is restricted at the AIS of most neurons (yellow arrows in panel $\mathbf{A}, \mathbf{B}$ ) or distributed all along the axon in a subpopulation of neurons (red arrows in panel $\mathbf{A}$ ) identified as GABAergic neurons using GAD65 labeling (C, red). (D) Granule cells positive for prox1 (blue asterisk) display Kv1.2 localized at the AIS. (E,F) GABAergic neurons including PV+ neurons (E, blue) exhibit high expression of Kv1.2 all along their axon and clusters of $\mathrm{Na}^{+}$channels labeled using anti-panNav mAb (F, red). (G,H) Fluorescence intensity profiles were generated starting from the AIS (G, white arrows) following the axon. The axonal clusters of ankyrinG are indicated with red arrows (G,H). Kv1.2 is co-localized with ankyrinG at the AIS, but do not co-cluster with ankyrinG along the axon. Note that Kv1.2 is restricted to the AlS (G, yellow arrow) in a neighboring neuron (asterisk). Scale bar: $50 \mu \mathrm{m}$ in panel (A); $20 \mu \mathrm{m}$ in panel (B-G). 

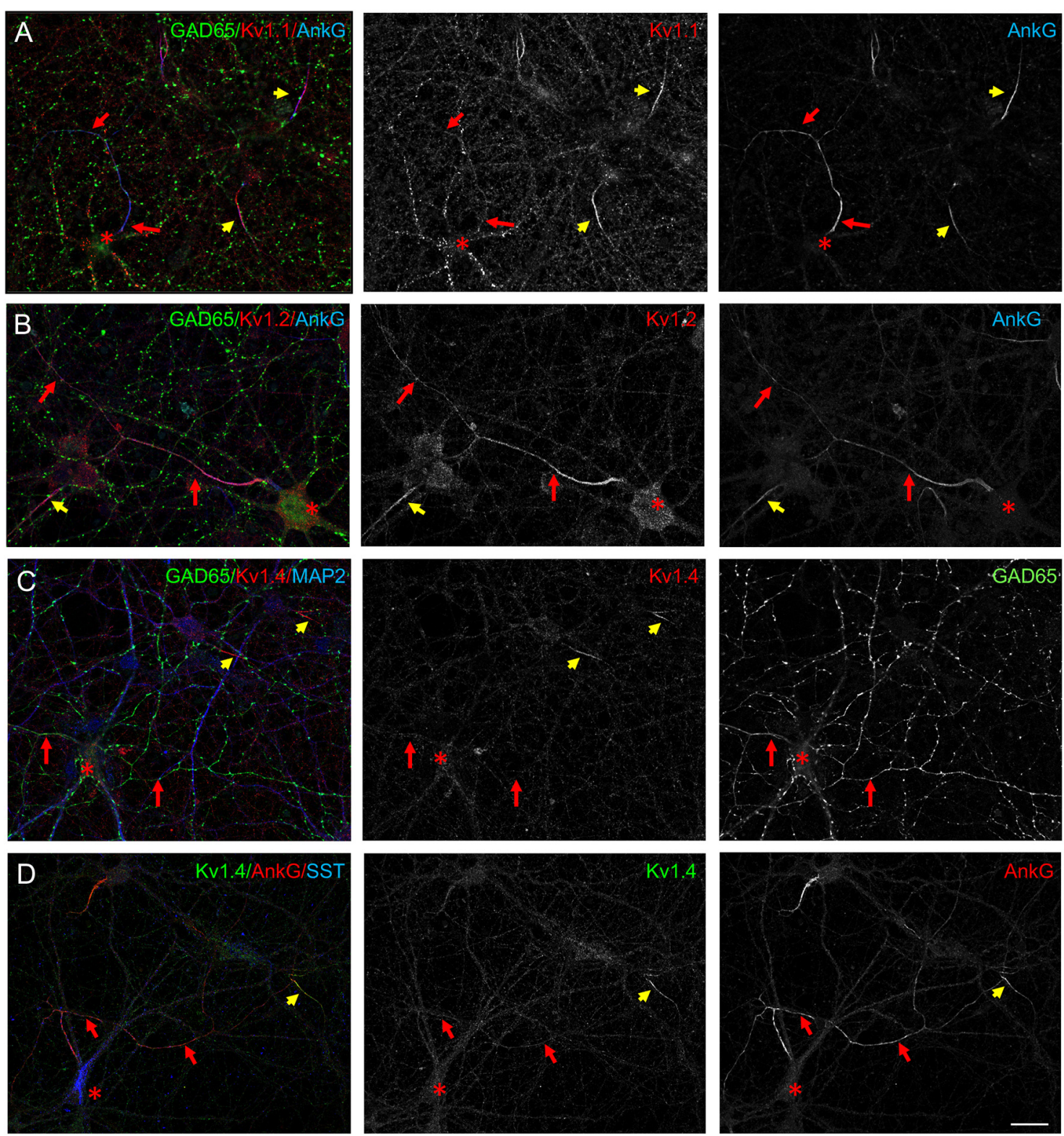

FIGURE 2 | Expression of Kv1 subunits in cultured hippocampal neurons. Cultured hippocampal neurons at DIV21 were immunostained for Kv1.1 (A), Kv1.2 (B), or Kv1.4 (C,D). Double-staining for GAD65 (A-C, green) or SST (D, blue) and ankyrinG (A-C, blue; D, red). Kv1.1, Kv1.2, and Kv1.4 are expressed at the AIS of excitatory neurons co-localized with ankyrinG (yellow arrows) and negative for SST or GAD65. (A) Kv1.1 is faintly detected along the axon (red arrows) and is present in intracellular vesicles in inhibitory neurons. (C,D) Kv1.4 is not detected along the inhibitory axons (red arrows). The cell bodies of SST- and GAD65-positive neurons are indicated with red asterisks. Scale bar: $20 \mu \mathrm{m}$.

illustrated by the plot profile (Figures $\mathbf{5 A , B , E , F ) . ~ S i n c e ~ p r o t e i n ~}$ $4.1 \mathrm{~B}$ is known to bind the cytoplasmic tail of Caspr2 and is required for the recruitment of the juxtaparanodal complex in myelinated fibers, we also analyzed its distribution and found that it was present along the distal axon and faintly expressed at the AIS of inhibitory neurons (Figures 5C,G). Similarly to TAG1 and Caspr2, ADAM22, another CAM associated with the Kv1 complex at the AIS (Ogawa et al., 2008; Hivert et al., 2019) was found to be preferentially expressed all along inhibitory axons in mature cultured hippocampal neurons (Figure 5D). ADAM22 appeared to be enriched at the AIS of inhibitory neurons, in a manner similar to TAG-1 (Figure 5D, red arrows).

\section{Interdependent Expression of Caspr2, TAG-1, and Protein 4.1B in Axons of SST and PV Neurons}

To determine whether the proteins of the Kv1 complex are interdependent for their enrichment along hippocampal inhibitory axons, we analyzed their expression taking advantage 

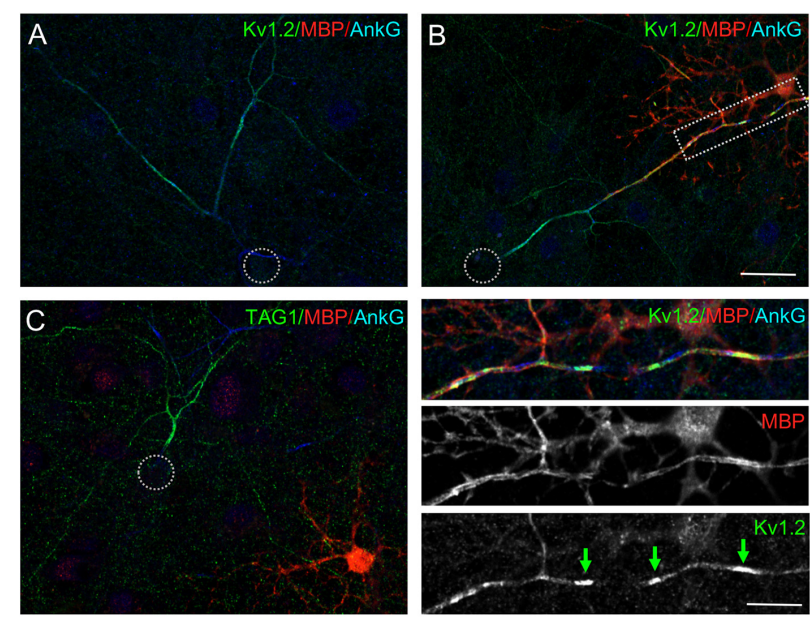

FIGURE 3 | Kv1.2 and TAG-1 are highly expressed along axons before myelination. Cultured hippocampal neurons at DIV21 were immunostained for Kv1.2 (A,B, green) or TAG-1 (C, green), MBP (red) as a marker of oligodendrocytes and ankyrinG (blue). (A,C) A number of unmyelinated neurons are labeled for Kv1.2 or TAG-1 all along their axons. (B) Few neurons are myelinated and show high density of Kv1.2 close to ankyrinG clusters under myelinated segments (green arrows in the inset). Scale bar: $20 \mu \mathrm{m} ; 10$ inset $\mu \mathrm{m}$.

of $\mathrm{KO}$ mice for TAG-1 or $4.1 \mathrm{~B}$ protein. Hippocampal cell cultures from wild type, TAG-1 KO and 4.1B KO mouse embryos were performed in parallel on the same day. As expected, we observed the absence of immunostaining for TAG-1 or $4.1 \mathrm{~B}$ in hippocampal neurons from TAG-1 or 4.1B $\mathrm{KO}$ mice, respectively, confirming the specificity of immunostaining in wild type neurons (Figures 6F,G, 7C). Live immunostaining at DIV21 indicated that the cell surface expression of Caspr2 was strongly altered in the inhibitory neurons from either TAG-1 or $4.1 \mathrm{~B} \mathrm{KO}$ mice, as shown for $\mathrm{SST}^{+}$neurons (Figures $6 \mathrm{~A}-\mathrm{C}$ ). Specifically, $92 \%$ of $\mathrm{SST}^{+}$neurons were positive for Caspr 2 in the wild type, whereas only $64 \%$ or $48 \%$ of them expressed Caspr 2 in the TAG-1 or 4.1B KO hippocampal cultures, respectively (Figure 6D). Similarly, only half of the $\mathrm{PV}^{+}$neurons expressed Caspr 2 in the TAG-1 and 4.1B KO (46 and 42\%, respectively) by comparison with the wild type cultures ( $82 \%$, Figure $6 \mathrm{E})$. These data indicate that TAG-1 and $4.1 \mathrm{~B}$ proteins are necessary for the proper expression of Caspr2 in inhibitory neurons, suggesting that Caspr2 may be stabilized in a preformed complex along the axonal membrane before myelination.

Next, we analyzed the axonal surface expression of TAG1 in hippocampal cultures from 4.1B KO mice (Figure 7). Remarkably, we observed that in some inhibitory neurons the protein was totally missing, whereas in other cells TAG1 expression was restricted to the AIS as shown for $\mathrm{SST}^{+}$ neurons (Figure 7B, red arrows). Precisely, TAG-1 expression was detected only at the AIS in $16.7 \%$ of the $\mathrm{SST}^{+}$neurons in the $4.1 \mathrm{~B} \mathrm{KO}$ versus $6 \%$ in wild type (Figure 7D). Likewise, TAG-1 was restricted at the AIS in $14.6 \%$ of the $\mathrm{PV}^{+}$neurons in $4.1 \mathrm{~B} \mathrm{KO}$ versus $2.7 \%$ in the wild-type (Figure $7 \mathrm{E}$ ). Since the axonal expression of Caspr2 is altered in the absence of protein
$4.1 \mathrm{~B}$, altogether these data suggest that the association of TAG1 in complex with Caspr2 and 4.1B may modulate the distal distribution of the protein along the inhibitory axons.

\section{Kv1.2 Distribution Is Not Altered in Knock-Out Mice for TAG-1 or Protein 4.1B}

The distribution of Kv1 channels at the juxtaparanodes is strongly altered in myelinated axons of the KO mice for TAG-1 or 4.1B (Traka et al., 2003; Cifuentes-Diaz et al., 2011; Hivert et al., 2016). It was therefore important to analyze whether the expression or distribution of Kv1.2 could be impaired at an early step in pre-myelinated GABAergic axons deficient for TAG-1 or 4.1B. We did not observe any difference in the expression of Kv1.2 either in $\mathrm{SST}^{+}$or $\mathrm{PV}^{+}$neurons deficient for TAG-1 or protein $4.1 \mathrm{~B}$, compared to the wild type situation as illustrated for $\mathrm{SST}^{+}$ neurons (Figures 8A-C). Kv1.2 was expressed in approximately $90 \%$ of the $\mathrm{SST}^{+}$and $\mathrm{PV}^{+}$neurons in the cultures from wild type, TAG-1 or 4.1B KO mice at DIV21 (Figures 8D,E). Furthermore, the high expression of Kv1.2 channels was correlated with the presence of ankyrinG clusters both in the wild type and mutant mice (Figures 8A-C). All together, these results point toward a mechanism for recruiting Kv1 channels along premyelinated inhibitory axons, which is independent from TAG-1 and protein $4.1 \mathrm{~B}$.

\section{In vivo Expression of the Cell Adhesion Molecules Associated With the Kv1 Channels in the Developing Hippocampus}

To evaluate the physiological relevance of our observations in vitro, we further addressed whether GABAergic neurons may also selectively express the Kv1 complex proteins in the developing hippocampus. The onset of myelination of GABAergic neurons in the hippocampus was reported from P14 (Freeman et al., 2015). Using immunostaining on hippocampal tissue sections from rats at post-natal day 21 (P21), we analyzed the expression of TAG-1 in SST ${ }^{+}$and the $\mathrm{PV}^{+}$inhibitory neurons (Figure 9). We observed that the $\mathrm{SST}^{+}$neurons located in the stratum oriens of the CA1 region were positive for TAG-1 $\left(42.1 \pm 4.3 \%, n=3\right.$ ) (Figures 9A,C). In contrast, $\mathrm{SST}^{+}$cells in other hippocampal regions, such as the hilus of the dentate gyrus, were negative for TAG-1 (Figures 9A,D). The $\mathrm{PV}^{+}$neurons expressed TAG-1 within the pyramidal layer $(69.9 \pm 8.3 \%, n=3)$ (Figures 9B,E) while they were negative in other areas, such as the stratum lacunosum moleculare (Figure 9F). In addition, these subtypes of inhibitory neurons were negative for TAG-1 within the other hippocampal areas i.e., the CA3 and the dentate gyrus (Figures 9A,B). We showed that TAG-1 was enriched at the AIS of $\mathrm{SST}^{+}$cells proximal to the myelin segment immunostained for MBP (Figure 9G) and also localized at the AIS of $\mathrm{PV}^{+}$cells using double-staining for ankyrinG (Figure 9H).

Next, we analyzed the pattern of expression of Kv1.2 in the CA1 region of the hippocampus (Figure 10). We found that Kv1.2 was localized at the AIS of $\mathrm{PV}^{+}$cells in the pyramidal layer (Figures $\mathbf{1 0 A}, \mathbf{A}$ ') and $\mathrm{SST}^{+}$cells in the stratum 

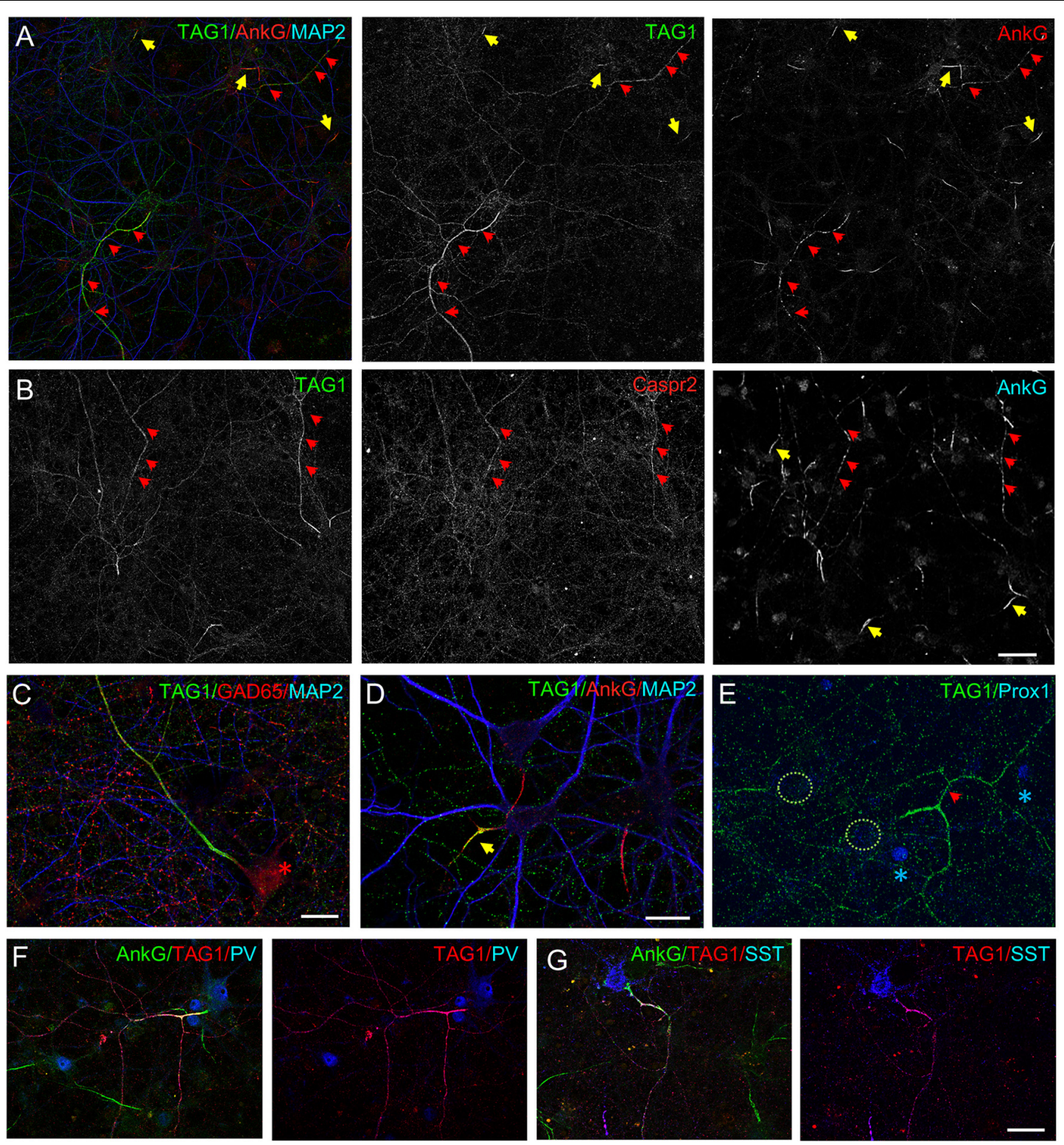

FIGURE 4 | Cell-type specific expression and distribution of TAG-1 and Caspr2 in cultured hippocampal neurons. Hippocampal neurons at DIV21 were surface labeled using anti-TAG-1 mAb (A, C-G) or double-labeled for TAG-1 and Caspr2 (B). Cells were fixed and permeabilized before immunostaining for intracellular markers. (A,B) In some neurons, TAG-1 and Caspr2 are expressed all along the axons which display clusters of ankyrinG (red arrows). TAG-1 is also restricted at the AIS in a subpopulation of neurons (yellow arrows in panel $\mathbf{A}$ ) as illustrated at high magnification (D) and is not expressed in granule cells labeled for prox1 (E, blue asterisks). TAG-1 is highly concentrated along the axon of GABAergic neurons (C, red asterisk) including PV-positive (F, blue) and SST-positive (G, blue) inhibitory neurons. Single confocal section (A-D) or maximum intensity of confocal images (4-Z steps of $440 \mathrm{~nm}$ ) in panel (F,G). Scale bar: $50 \mu \mathrm{m}$ in panel (A,B); $20 \mu \mathrm{m}$ in panel (C-G).

pyramidale (Figure 10C) and oriens (Figure 10B), but distally as compared to ankyring (Figure $\mathbf{1 0 B} /$ ). We observed that $\mathrm{PV}^{+}$cells in the pyramidal layer (Figure 10D) and $\mathrm{SST}^{+}$cells located in the stratum oriens (Figure 10E) were positive for both Caspr2 and Kv1.2.

Our results show that the Kv1 complex proteins are enriched in the GABAergic neurons of the CA1 hippocampal region in vivo. Both PV and SST inhibitory neurons specifically express Kv1.2, TAG-1, and Caspr2, similarly to our in vitro findings.
Moreover, a layer-specific expression of TAG-1 can be observed for SST and PV neurons that may indicate a specialization of these cells associated with myelination.

\section{DISCUSSION}

The Kv1 channels play a prominent role in repolarising the axon after action potential initiation at the AIS and 


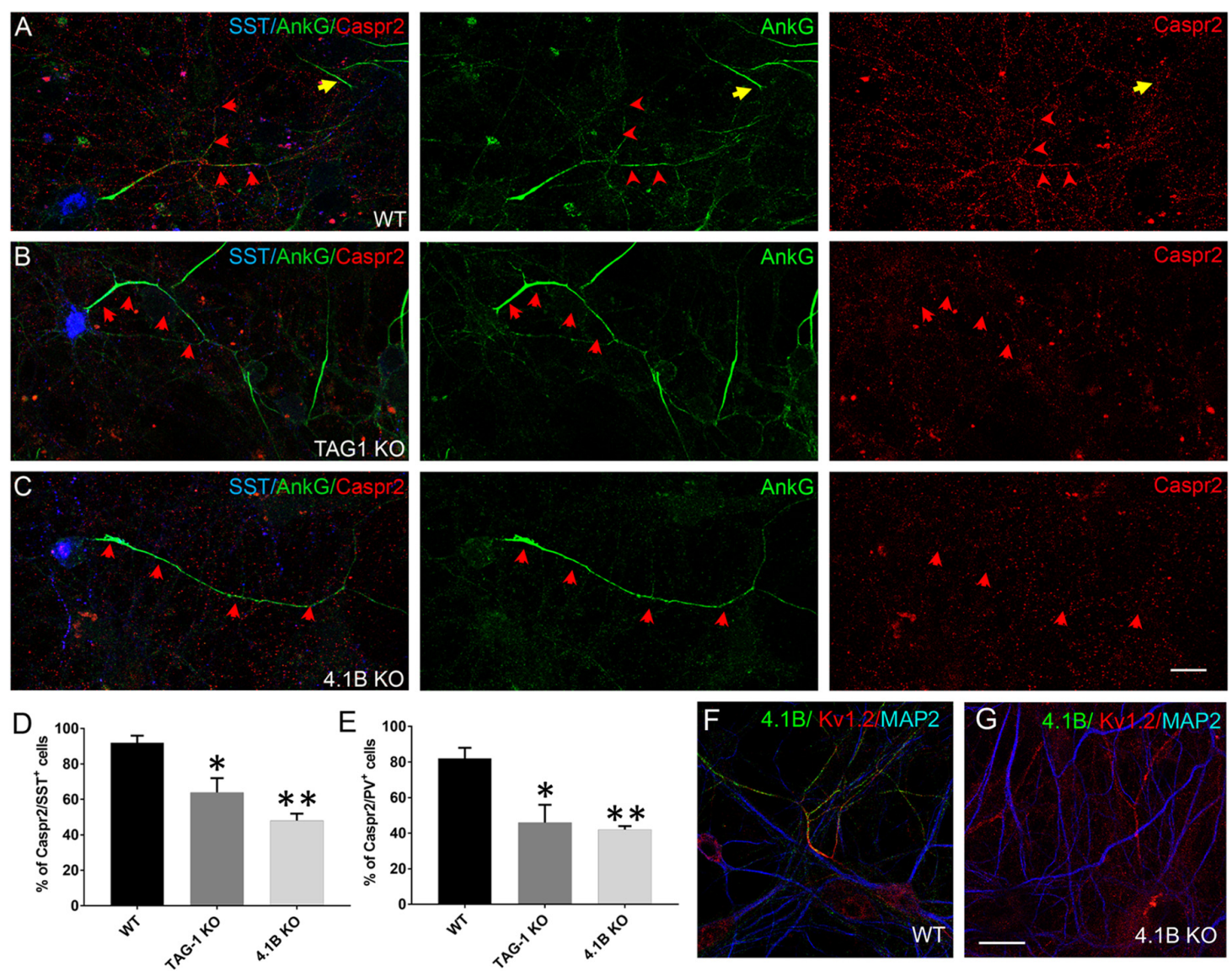

FIGURE 6 | Caspr2 expression is disorganized in inhibitory neurons from TAG-1 or 4.1B KO mice. (A-C) Hippocampal neurons from wild type (WT) (A), TAG-1 (B), or 4.1B KO mice (C) at DIV21 were surface labeled with human anti-Caspr2 antibodies (red). Cells were then fixed, permeabilized and immunostained with mouse anti-ankyrinG (green) and goat anti-SST (blue) antibodies. Axons of SST ${ }^{+}$cells are indicated with red arrows. The AIS of one SST-negative cell is indicated with a

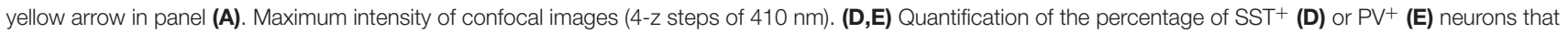
were labeled for Caspr2. The percentage of neurons showing Caspr2 axonal staining is significantly reduced in cells from either TAG-1 or 4.1B KO mice.

Mean \pm SEM of three independent experiments. For each experiment, at least 50 neurons were analyzed. For the statistics, one-way ANOVA with post hoc Dunnett test was used. Comparison with wild type group: ${ }^{*} p<0.05$ and ${ }^{* *} p<0.01$. (F,G) Neurons from wild type (F) or $4.1 \mathrm{~B}$ KO (G) mice immunostained using rabbit anti-4.1B (green), chicken anti-MAP2 (blue), and mouse anti-Kv1.2 (red) antibodies. Scale bar: $20 \mu \mathrm{m}$.

in regulating propagation at the juxtaparanodes (Trimmer, 2015). During myelination, these channels are progressively enriched at the juxtaparanodes and at early stages, when the paranodal junctions have not yet formed or stabilized, Kv1 transiently localizes at nodes and paranodes (Vabnick et al., 1999; Hivert et al., 2016) and may be directly involved in speeding repolarisation to allow trains of action potentials. The dynamic distribution and precise sub-compartmental profile of $\mathrm{Kv} 1$ is thought to play an essential role in the developing axons switching from a continuous to a saltatory mode of conduction. In the present study, we show that in hippocampal cell cultures, the Kv1.2 subunits are selectively expressed all along GABAergic axons including the AIS before myelination, together with clusters of Nav1 channels and ankyrinG. Inhibitory axons are also highly enriched in molecules originally identified as part of the Kv1 complex at the juxtaparanodal regions of myelinated fibers, including the CAMs TAG-1, Caspr2, and ADAM22, as well as the scaffolding protein 4.1B. Cultures from TAG-1- or protein $4.1 \mathrm{~B}$-deficient mice indicate that the expression of TAG-1, Caspr2 and protein $4.1 \mathrm{~B}$ is interdependent whereas the distal distribution of the Kv1.2 subunits is maintained in the absence of TAG-1 or protein 4.1B. In vivo, only subsets of SST and PV GABAergic neurons are positive for TAG-1 in the juvenile rat hippocampus, including the SST cells in the stratum oriens and the PV neurons in the stratum pyramidale, which also express Kv1.2 channels. This accurate distribution of ion channels and associated molecules along pre-myelinated axons may be crucial to regulate firing during development.

\section{Axonal Distribution of the Kv1 Complex in Inhibitory Hippocampal Neurons}

In myelinated axons, the proper distribution of the Nav1 and Kv1 channels is strongly dependent on CAMs at nodal, 

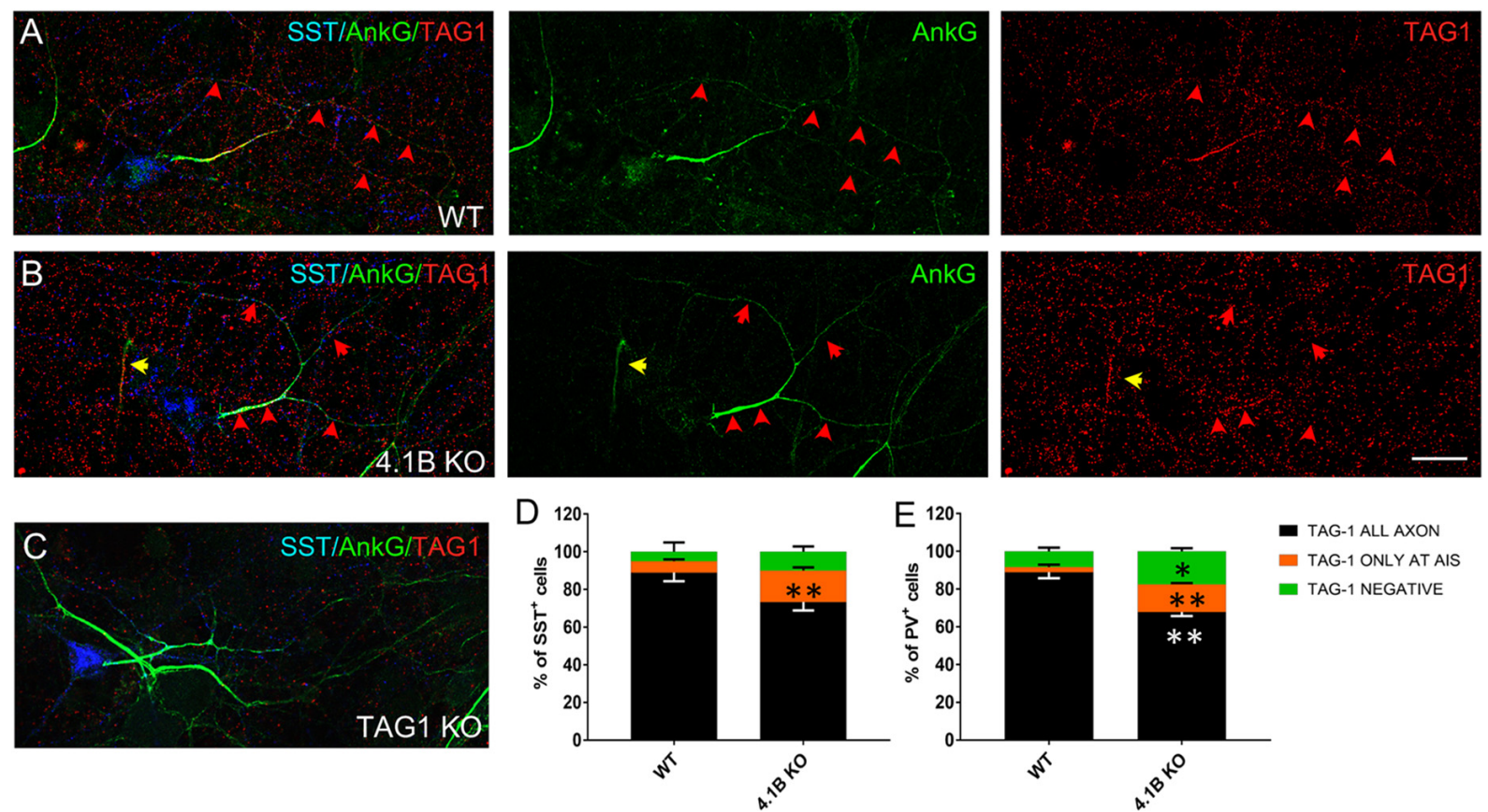

- TAG-1 ALL AXON

- TAG-1 ONLY AT AIS

- TAG-1 NEGATIVE

FIGURE 7 | TAG-1 displays an abnormal distribution in inhibitory neurons from 4.1B KO mice. (A-C) Hippocampal neurons from wild type (A), 4.1B KO (B), or TAG-1 KO (C) mice were surface labeled at DIV21 for TAG-1 (red). Cells were fixed, permeabilized and immunostained with mouse anti-ankyrinG (green) and goat anti-SST (blue) antibodies. TAG-1 is enriched all along the axon in wild type inhibitory neurons (A), while it is mainly expressed at the AIS in cells derived from 4.1B $\mathrm{KO}$ mice (B). The AIS of one SST-negative cell is indicated with a yellow arrow in panel (B). Note that TAG-1 immunostaining is reduced to background in neurons from TAG-1 KO mice (C). Maximum intensity of confocal images (4-z steps of $410 \mathrm{~nm}$ ). (D,E) Quantification of the percentage of SST+ $(\mathbf{D})$ or PV+ (E) neurons showing TAG-1 all along the axon (black), only at the AIS (restricted to a distance of $50 \mu \mathrm{m}$ from the soma, orange) or completely absent (green). Mean \pm SEM of three independent experiments. For each experiment, at least 50 neurons were analyzed. For statistics, the paired $t$-test was used. Comparison with wild type group: ${ }^{*} p<0.05$ and ${ }^{* *} p<0.01$. Scale bar: $20 \mu \mathrm{m}$.

paranodal, and juxtaparanodal regions. Specifically, a complex of TAG-1, Caspr2, and protein 4.1B mediates axo-glial contacts at juxtaparanodes and is required for $\mathrm{Kvl}$ channel clustering (Poliak et al., 2003; Traka et al., 2003). However, TAG1 and Caspr2 are dispensable for the trapping of the Kv1 channels at the AIS (Ogawa et al., 2008; Duflocq et al., 2011). Here, we observed that TAG-1 and ADAM 22 are enriched at the AIS and expressed all along pre-myelinated inhibitory axons, whereas Caspr2 and protein $4.1 \mathrm{~B}$ display a more distal distribution. Furthermore, taking advantage of $\mathrm{KO}$ mouse lines, we showed that Caspr2, TAG-1, and $4.1 \mathrm{~B}$ are interdependent for their distribution along inhibitory axons. The expression of Caspr2 was strongly reduced along the inhibitory axons from TAG-1 or 4.1B KO hippocampal cultures. TAG1 and protein $4.1 \mathrm{~B}$ bind the ectodomain and the cytoplasmic tail of Caspr2, respectively, and may be required for its stabilization at the axonal membrane. We hypothesize that the association of Caspr2 with protein $4.1 \mathrm{~B}$ may stabilize Caspr2 at the axolemma by inhibiting its internalization (Bel et al., 2009; Pinatel et al., 2017). Next, we observed that in contrast to Caspr2, the distal distribution of TAG-1 in inhibitory axons was reduced only in a small percentage of $4.1 \mathrm{~B}$-deficient neurons. We previously reported the level of TAG-1 is not altered in the brain of $4.1 \mathrm{~B} \mathrm{KO}$ mice (Cifuentes-Diaz et al., 2011). TAG-1 is trapped at the AIS independently of Caspr2 which is not enriched at that site. Our data suggest that TAG-1 may be associated with ADAM22 at the AIS and also along the axon. Indeed, we recently reported that TAG-1 can be associated with ADAM22 using co-immunoprecipitation experiments and that the two CAMs are sorted together in axonal transport vesicles (Hivert et al., 2019). However, the persistence of Kv1.2 subunits along the axons in TAG-1- and protein 4.1B-deficient cells reveals that the Caspr2/TAG-1/4.1B complex may be dispensable for the distal distribution of Kv1 channels. This indicates that the Kv1.2 tetramers which are axonally transported with their Kvß2 accessory subunits ( $\mathrm{Gu}$ and $\mathrm{Gu}, 2010)$ are targeted to the axon independently from their associated CAMs. In the same way, the CAM neurofascin186, which is implicated in the initial nodal clustering of Nav1 channels in myelinated axons, is targeted at the axonal membrane through distinct mechanisms than the Nav1 channels (Zhang et al., 2012). In addition, neurofascin 186 is not required for the clustering of pre-nodal complexes in pre-myelinated inhibitory neurons (Dr. Nathalie Sol-Foulon, personal communication). This early clustering of Nav1 channels linked with ankyrinG appears to be mediated by extracellular matrix proteins and soluble form of CAMs secreted by oligodendrocytes and it does not require the contact with the glial membrane. Our results indicate that this is unlikely to be the case for the clustering 

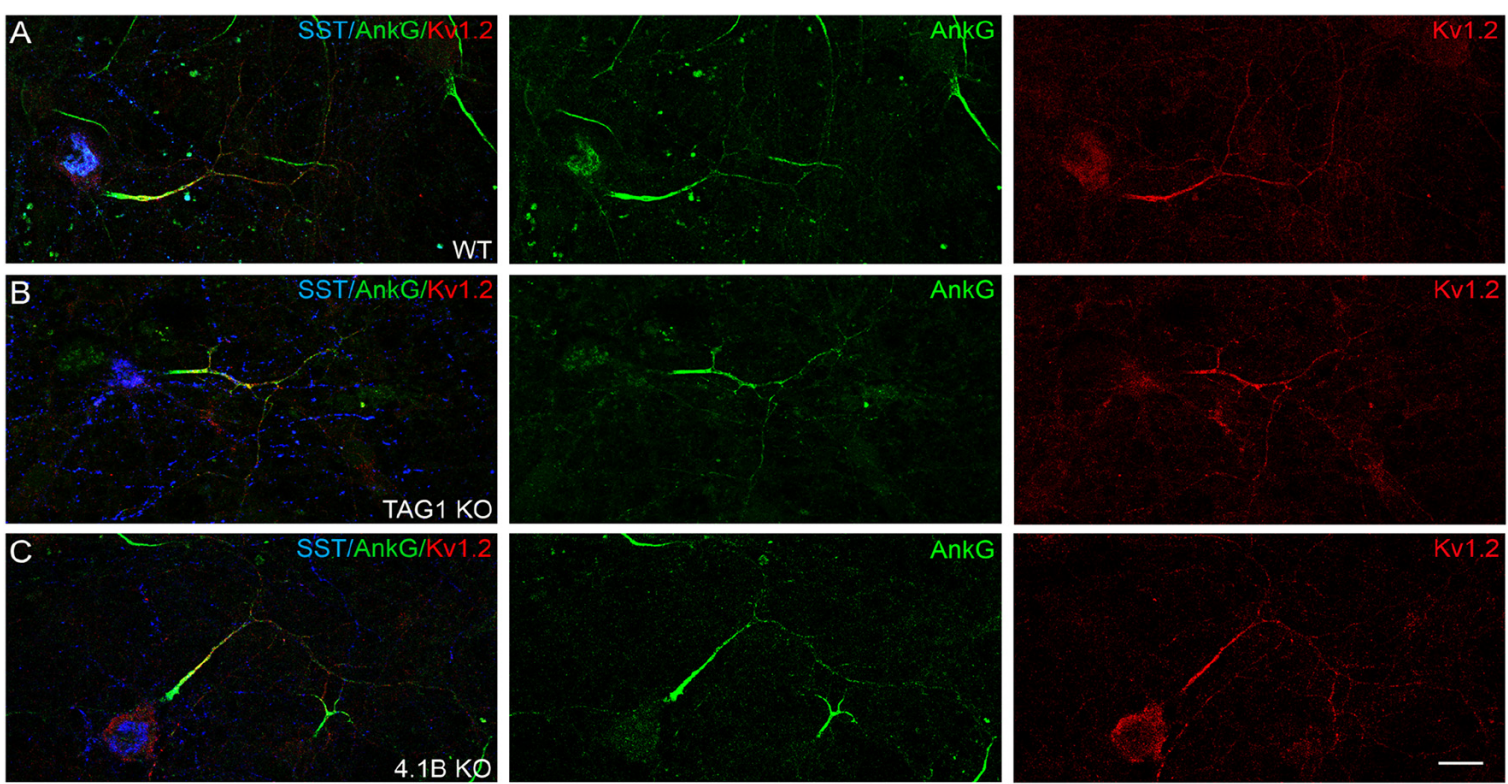

D

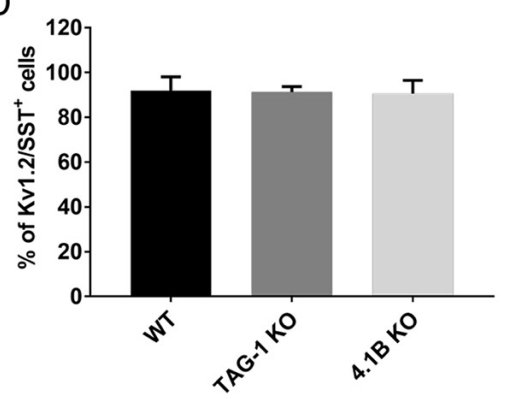

E

FIGURE 8 | The distribution of Kv1.2 is not altered in inhibitory neurons from TAG-1 or 4.1B KO mice. (A-C) Hippocampal neurons at DIV21 immunostained fo ankyrinG (green), Kv1.2 (red), and SST (blue). Kv1.2 is expressed all along the axons of inhibitory neurons from wild type (A), TAG-1 (B), or 4.1B KO mice (C). Maximum intensity of confocal images (4-z steps of $410 \mathrm{~nm}$ ). (D-E) Quantification of inhibitory neurons showing double-staining for SST (D) or PV (E) and Kv1.2. Mean \pm SEM of three independent experiments. For each experiment, at least 50 neurons were analyzed. For the statistics, one-way ANOVA with post hoc Dunnett test was used and no statistical significance was found. Scale bar: $20 \mu \mathrm{m}$.

of the Kv1 channels at the nodes since we observed that the Kv1 channels are enriched at the AIS and thereafter uniformly distributed along the pre-myelinated axon, with the clustering of Kv1 being only detected where the myelin segments contact the axon. Interestingly, such a contact-dependent clustering of Kv1 channels has also been reported using cocultures of hippocampal neurons with TAG-1 expressing HEK cells ( $\mathrm{Gu}$ and $\mathrm{Gu}, 2011$ ).

We further show that both the Kv1.1 and Kv1.2 subunits are expressed in GABAergic neurons whereas the Kv1.4 subunit is only localized at the AIS of excitatory neurons in hippocampal cell culture. However, Kv1.1 is faintly expressed along the inhibitory axons while it is strongly detected as intracellular vesicles. In this context, it is interesting to note that Kv1.4, which contains an ER export signal, has been shown to induce the cell surface targeting of Kv1.1 (Manganas and Trimmer, 2000; Lai and Jan, 2006; Jenkins et al., 2011). Therefore, these results suggest that Kv1 channels in inhibitory axons in hippocampal cell culture may mainly consist of Kv1.2 subunits. Importantly, the different composition of Kv1 tetramers allows distinct thresholds of channel activation (Bagchi et al., 2014).

\section{In vivo Selective Expression of TAG-1 in Subtypes of GABAergic Neurons in the Hippocampus}

Recent reports have highlighted the possibility that different subtypes of GABAergic neurons could be myelinated. Longrange projecting inhibitory neurons connecting hippocampus with extra-hippocampal areas are known to be myelinated (Jinno et al., 2007; Melzer et al., 2012). This is the case of SST neurons in the stratum oriens of CA1, which project to the subiculum and to the entorhinal cortex. Surprisingly, it has been recently reported that a substantial fraction of myelin, both in mouse and human neocortex, belongs to GABAergic inhibitory neurons, in particular fast-spiking PV interneurons (Micheva et al., 2016; Stedehouder et al., 2017). The PV cells in the CA1 

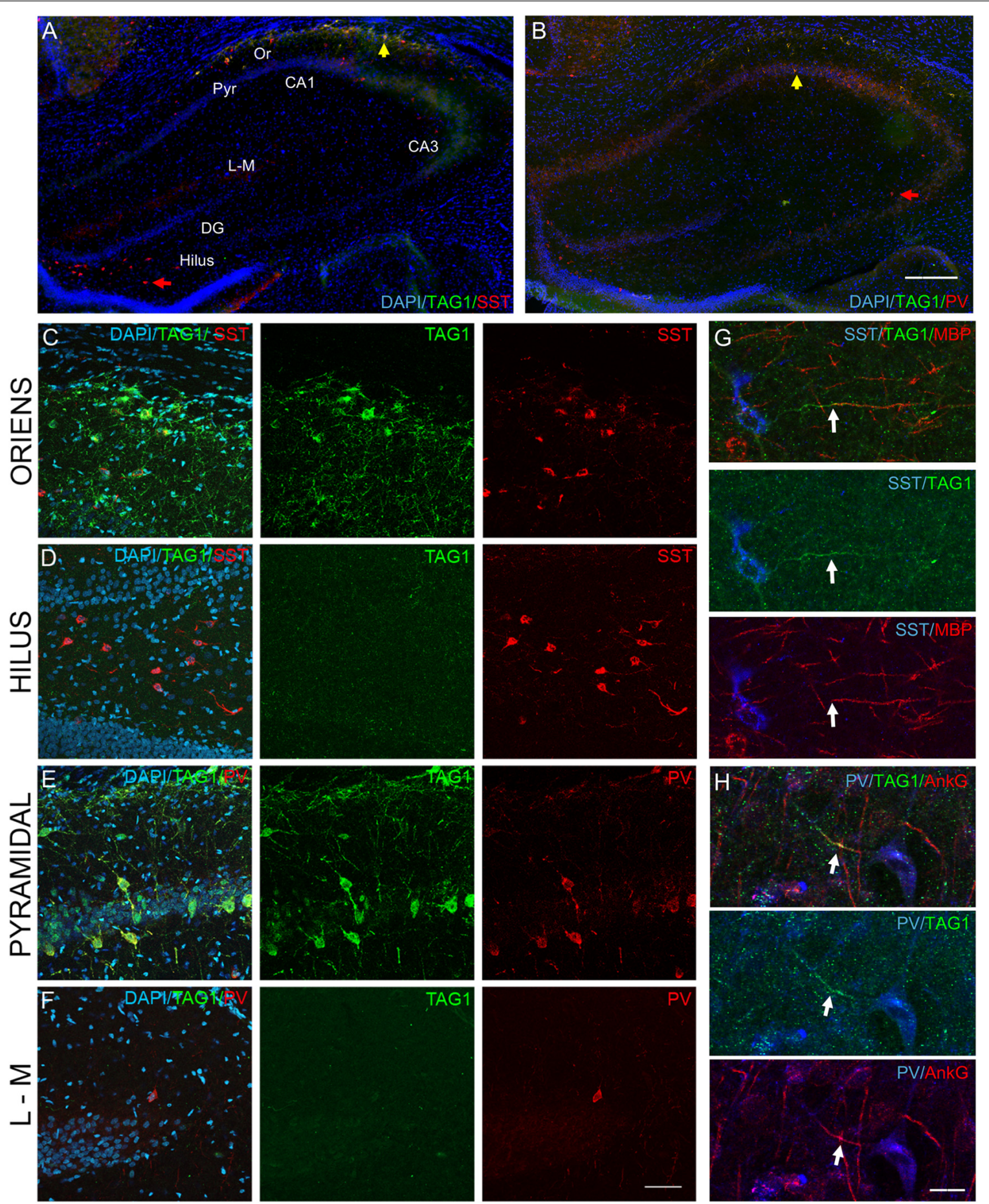

FIGURE 9 | TAG-1 is expressed in subtypes of $\mathrm{PV}^{+}$and $\mathrm{SST}^{+}$inhibitory neurons in the hippocampus. (A,B) Coronal hippocampal cryosections from postnatal day 21 (P21) rat at low magnification, stained for the nuclear marker DAPI, TAG-1 (green) and SST (A) or PV (B). The indicated hippocampal areas, i.e., the stratum oriens (Or), the pyramidal layer (Pyr), the stratum lacunosum-moleculare (L-M) and the hilus, are shown at high magnification in panel (C-F). (C-F) Coronal hippocampal cryosections from P21 rat labeled for TAG-1 (green), SST (red, C,D) or PV (red, E,F), and DAPI. TAG-1 is selectively expressed by SST ${ }^{+}$and PV ${ }^{+}$ inhibitory neurons in specific layers of the CA1 hippocampus, the stratum oriens for SST+ neurons (C and yellow arrow in panel $\mathbf{A}$ ) and the pyramidal layer for $\mathrm{PV}{ }^{+}$ cells ( $\mathbf{E}$ and yellow arrow in panel B). TAG-1 is not expressed in other areas of the hippocampus, such as in the SST ${ }^{+}$cells in the hilus of the dentate gyrus (D and red arrow in panel $\mathbf{A}$ ) or the $\mathrm{PV}^{+}$cells in the stratum lacunosum-moleculare (F) or CA3 pyramidal layer (red arrow in panel $\left.\mathbf{B}\right)$. (G,H) Sagittal hippocampal cryosections from P21 rat labeled for SST, TAG-1, and MBP as a marker of myelin (G) or PV, TAG-1, and ankyrinG (H). TAG-1 is localized at the AIS (white arrows) in both $\mathrm{SST}^{+}$and $\mathrm{PV}^{+}$inhibitory neurons in the $\mathrm{CA} 1$ hippocampal region. Note that the $\mathrm{SST}^{+}$neuron positive for TAG-1 is myelinated. Wide-field $(\mathbf{A}, \mathbf{B})$ and confocal (C-H) microscopy images. Maximum intensity of 6-z steps of $830 \mathrm{~nm}(\mathbf{C}-\mathbf{F})$ or 3-z steps of $1.38 \mu \mathrm{m}$ (G) or $1 \mu \mathrm{m}$ (H). Scale bar: $80 \mu \mathrm{m}$ in panel (A,B); $50 \mu \mathrm{m}$ in panel (C-F); $15 \mu \mathrm{m}$ in panel (G,H). 

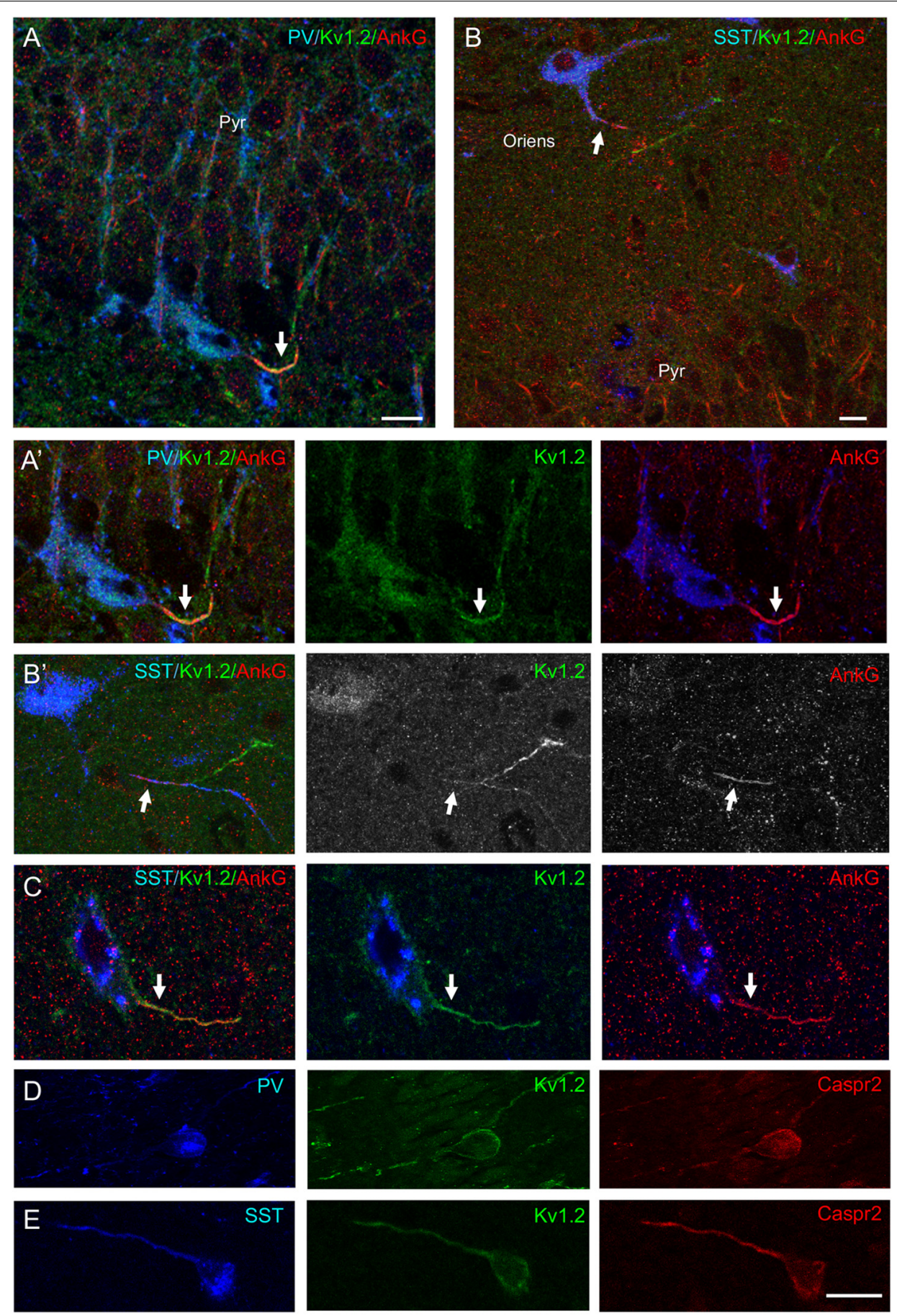

FIGURE $10 \mid \mathrm{KV} 1.2$ is expressed at the AIS of $\mathrm{PV}^{+}$and $\mathrm{SST}^{+}$inhibitory neurons in the CA1 layer of the rat hippocampus. Sagittal hippocampal cryosections from P21 rat labeled for PV (A, blue) or SST (B,C, blue), ankyrinG (red) or Caspr2 (red) and Kv1.2 (green). (A,A $)$ Kv1.2 is detected at the AlS of a PV+ cell in the stratum pyramidale (Pyr). (B-C) Kv1.2 is localized at the AIS of SST ${ }^{+}$neurons in the stratum oriens (B,B') and stratum pyramidale (C). AlS are indicated with arrows. Note that $\mathrm{Kv1} .2$ is distributed more distally than ankyrinG along the axon (B/). (D,E) Caspr2 and Kv1.2 are co-expressed in PV ${ }^{+}$cells of the stratum pyramidale (D) and $\mathrm{SST}^{+}$cells in the stratum oriens (E). Single confocal section (A-C) and maximum intensity of 5-Z steps of $1.31 \mu \mathrm{m}$ (insets in panel $\mathbf{A} \mathbf{\prime}, \mathbf{B}$ ') or $4-\mathbf{z}$ steps of $830 \mathrm{~nm}$ (D,E). Scale bar: $15 \mu \mathrm{m}$.

stratum pyramidale of hippocampus have been described to be frequently myelinated at 8-12 weeks of age, mainly on the proximal axonal segments, independently of their morphological subtypes (i.e., basket or bi-stratified) (Stedehouder et al., 2017). PV interneurons are high energy demanding cells, for which it is logical to think that myelin may provide axonal metabolic 
support (Kann et al., 2014; Krasnow and Attwell, 2016). Our results indicate that TAG-1 is exclusively expressed by some subtypes of GABAergic neurons in the rodent hippocampus, namely the PV cells of the pyramidal layer and the SST neurons of the stratum oriens in 3 -week old rats. It remains to be precisely determined whether this selective expression of TAG-1 as a juxtaparanodal component may be associated with the myelinated fate of both local PV interneurons in the stratum pyramidale and long-range projecting SST neurons of the stratum oriens. Supporting this possibility, we observed that TAG-1 is enriched at the AIS of some $\mathrm{SST}^{+}$cells of the stratum oriens, proximal to myelin segments.

The inhibitory axons of the PV and SST cells display prenodal clusters of Nav1 channels, which have been shown to promote acceleration of conduction in pre-myelinated axons as analyzed in cultured hippocampal neurons (Freeman et al., 2015). The presence of Nav1 clusters along pre-myelinated PV interneurons as observed in vitro and also in vivo (Freeman et al., 2015) may be related with the results of $\mathrm{Hu}$ and Jonas (2014), which showed a gradual increase of $\mathrm{Na}^{+}$conductance in the distal axon of PV interneurons. The authors suggest that a high density of $\mathrm{Na}^{+}$channels could be necessary for ensuring both speed of propagation and fast-spiking action potentials in unmyelinated axons. In addition, it has been shown that Kv1.1 channels localized at the AIS dampen excitability and prevent high frequency discharge at normal subthreshold levels in fastspiking GABAergic cortical neurons (Goldberg et al., 2008). The Kv1.1 subunit is co-localized with ankyrinG at the AIS of PV basket cells in the hippocampus in vivo (Campanac et al., 2013). Here, we observed that the Kv1.2 subunit and TAG-1 are expressed at the AIS of both in PV and SST cells in the CA1 region of the hippocampus. Our data show the expression of a high density of Kv1 channels associated with CAMs occurring along pre-myelinated axons of subtypes of PV interneurons during development. With this respect, it will be important to analyze the physiological role of the high axonal content of the Kv1 complex in PV interneurons before myelination or in demyelinated pathological conditions.

\section{DATA AVAILABILITY}

All datasets generated for this study are included in the manuscript and/or the Supplementary Files.

\section{REFERENCES}

Bagchi, B., Al-Sabi, A., Kaza, S., Scholz, D., O’leary, V. B., Dolly, J. O., et al. (2014). Disruption of myelin leads to ectopic expression of $K(V) 1.1$ channels with abnormal conductivity of optic nerve axons in a cuprizone-induced model of demyelination. PLoS One 9:e87736. doi: 10.1371/journal.pone. 0087736

Bel, C., Oguievetskaia, K., Pitaval, C., Goutebroze, L., and Faivre-Sarrailh, C. (2009). Axonal targeting of Caspr2 in hippocampal neurons via selective somatodendritic endocytosis. J. Cell Sci. 122, 3403-3413. doi: 10.1242/jcs. 050526

Bhat, M. A., Rios, J. C., Lu, Y., Garcia-Fresco, G. P., Ching, W., St Martin, M., et al. (2001). Axon-glia interactions and the domain organization of myelinated

\section{ETHICS STATEMENT}

This study was carried out in accordance with the recommendations of the European and Institutional guidelines for the care and use of laboratory animals and approved by the local authority (laboratory's agreement number D13-055-8, Préfecture des Bouches du Rhône).

\section{AUTHOR CONTRIBUTIONS}

GB conceived and performed the experiments, analyzed the data, and wrote the manuscript. BH conceived and performed the experiments and analyzed the data. LG and DK provided the reagents and $\mathrm{KO}$ mice and discussed the data. VC discussed the data and provided the financial support. CF-S conceived and performed the experiments, analyzed the data, wrote the manuscript, provided the financial support, and supervised the study.

\section{FUNDING}

This work was supported by the Association pour la Recherche sur la Sclérose en Plaques (ARSEP) to CF-S and DK. GB is a postdoctoral fellow with the financial support of ARSEP. The project funded was entitled "Assembly of the juxtaparanodal complex under normal and pathological conditions."

\section{ACKNOWLEDGMENTS}

We are grateful to Drs. Oussama El Far, Eric Di Pasquale, Nathalie Sol-Foulon, and Agnès Baude for their helpful discussions. We thank the University of California, Davis/National Institutes of Health NeuroMab Facility and Developmental Studies Hybridoma Bank of the University of Iowa.

\section{SUPPLEMENTARY MATERIAL}

The Supplementary Material for this article can be found online at: https://www.frontiersin.org/articles/10.3389/fncel. 2019.00222/full\#supplementary-material

axons requires neurexin IV/Caspr/Paranodin. Neuron 30, 369-383. doi: 10. 1016/s0896-6273(01)00294-x

Buttermore, E. D., Dupree, J. L., Cheng, J., An, X., Tessarollo, L., and Bhat, M. A. (2011). The cytoskeletal adaptor protein band $4.1 \mathrm{~B}$ is required for the maintenance of paranodal axoglial septate junctions in myelinated axons. J. Neurosci. 31, 8013-8024. doi: 10.1523/JNEUROSCI.1015-11.2011

Buttiglione, M., Revest, J. M., Pavlou, O., Karagogeos, D., Furley, A., Rougon, G., et al. (1998). A functional interaction between the neuronal adhesion molecules TAG-1 and F3 modulates neurite outgrowth and fasciculation of cerebellar granule cells. J. Neurosci. 18, 6853-6870. doi: 10.1523/jneurosci.18-17-06853. 1998

Campanac, E., Gasselin, C., Baude, A., Rama, S., Ankri, N., and Debanne, D. (2013). Enhanced intrinsic excitability in basket cells maintains excitatory-inhibitory 
balance in hippocampal circuits. Neuron 77, 712-722. doi: 10.1016/j.neuron. 2012.12.020

Charles, P., Tait, S., Faivre-Sarrailh, C., Barbin, G., Gunn-Moore, F., DenisenkoNehrbass, N., et al. (2002). Neurofascin is a glial receptor for the paranodin/Caspr-contactin axonal complex at the axoglial junction. Curr. Biol. 12, 217-220. doi: 10.1016/s0960-9822(01)00680-7

Ching, W., Zanazzi, G., Levinson, S. R., and Salzer, J. L. (1999). Clustering of neuronal sodium channels requires contact with myelinating schwann cells. J. Neurocytol. 28, 295-301.

Cifuentes-Diaz, C., Chareyre, F., Garcia, M., Devaux, J., Carnaud, M., Levasseur, G., et al. (2011). Protein 4.1B contributes to the organization of peripheral myelinated axons. PLoS One 6:e25043. doi: 10.1371/journal.pone.0025043

Denisenko-Nehrbass, N., Oguievetskaia, K., Goutebroze, L., Galvez, T., Yamakawa, H., Ohara, O., et al. (2003). Protein 4.1B associates with both Caspr/paranodin and Caspr2 at paranodes and juxtaparanodes of myelinated fibres. Eur. J. Neurosci. 17, 411-416. doi: 10.1046/j.1460-9568.2003.02441.x

Devaux, J., and Gow, A. (2008). Tight junctions potentiate the insulative properties of small CNS myelinated axons. J. Cell Biol. 183, 909-921. doi: 10.1083/jcb. 200808034

Duflocq, A., Chareyre, F., Giovannini, M., Couraud, F., and Davenne, M. (2011). Characterization of the axon initial segment (AIS) of motor neurons and identification of a para-AIS and a juxtapara-AIS, organized by protein 4.1B. BMC Biol. 9:66. doi: 10.1186/1741-7007-9-66

Einheber, S., Meng, X., Rubin, M., Lam, I., Mohandas, N., An, X., et al. (2013). The 4.1B cytoskeletal protein regulates the domain organization and sheath thickness of myelinated axons. Glia 61, 240-253. doi: 10.1002/glia.22430

Eshed-Eisenbach, Y., and Peles, E. (2013). The making of a node: a co-production of neurons and glia. Curr. Opin. Neurobiol. 23, 1049-1056. doi: 10.1016/j.conb. 2013.06.003

Feinberg, K., Eshed-Eisenbach, Y., Frechter, S., Amor, V., Salomon, D., Sabanay, H., et al. (2010). A glial signal consisting of gliomedin and NrCAM clusters axonal $\mathrm{Na}+$ channels during the formation of nodes of ranvier. Neuron $65,490-502$. doi: 10.1016/j.neuron.2010.02.004

Freeman, S. A., Desmazieres, A., Simonnet, J., Gatta, M., Pfeiffer, F., Aigrot, M. S., et al. (2015). Acceleration of conduction velocity linked to clustering of nodal components precedes myelination. Proc. Natl. Acad. Sci. U.S.A. 112, E321-E328. doi: 10.1073/pnas.1419099112

Goldberg, E. M., Clark, B. D., Zagha, E., Nahmani, M., Erisir, A., and Rudy, B. (2008). K+ channels at the axon initial segment dampen near-threshold excitability of neocortical fast-spiking GABAergic interneurons. Neuron 58, 387-400. doi: 10.1016/j.neuron.2008.03.003

$\mathrm{Gu}, \mathrm{C}$., and Gu, Y. (2011). Clustering and activity tuning of Kv1 channels in myelinated hippocampal axons. J. Biol. Chem. 286, 25835-25847. doi: 10.1074/ jbc.M111.219113

Gu, Y., and Gu, C. (2010). Dynamics of Kv1 channel transport in axons. PLoS One 5:e11931. doi: 10.1371/journal.pone.0011931

Hivert, B., Marien, L., Agbam, K. N., and Faivre-Sarrailh, C. (2019). ADAM22 and ADAM23 modulate the targeting of the Kv1 channel-associated protein LGI1 to the axon initial segment. J. Cell Sci. 132:jcs219774. doi: 10.1242/jcs. 219774

Hivert, B., Pinatel, D., Labasque, M., Tricaud, N., Goutebroze, L., and FaivreSarrailh, C. (2016). Assembly of juxtaparanodes in myelinating DRG culture: differential clustering of the Kv1/Caspr2 complex and scaffolding protein 4.1B. Glia 64, 840-852. doi: 10.1002/glia.22968

Horresh, I., Bar, V., Kissil, J. L., and Peles, E. (2010). Organization of myelinated axons by Caspr and Caspr2 requires the cytoskeletal adapter protein $4.1 \mathrm{~B}$. J. Neurosci. 30, 2480-2489. doi: 10.1523/JNEUROSCI.5225-09.2010

Horresh, I., Poliak, S., Grant, S., Bredt, D., Rasband, M. N., and Peles, E. (2008), Multiple molecular interactions determine the clustering of Caspr2 and Kv1 channels in myelinated axons. J. Neurosci. 28, 14213-14222. doi: 10.1523/ JNEUROSCI.3398-08.2008

$\mathrm{Hu}, \mathrm{H}$., and Jonas, P. (2014). A supercritical density of $\mathrm{Na}(+)$ channels ensures fast signaling in GABAergic interneuron axons. Nat. Neurosci. 17, 686-693. doi: $10.1038 / \mathrm{nn} .3678$

Jenkins, P. M., Mcintyre, J. C., Zhang, L., Anantharam, A., Vesely, E. D., Arendt, K. L., et al. (2011). Subunit-dependent axonal trafficking of distinct alpha heteromeric potassium channel complexes. J. Neurosci. 31, 13224-13235. doi: 10.1523/JNEUROSCI.0976-11.2011
Jinno, S., Klausberger, T., Marton, L. F., Dalezios, Y., Roberts, J. D., Fuentealba, P., et al. (2007). Neuronal diversity in GABAergic long-range projections from the hippocampus. J. Neurosci. 27, 8790-8804. doi: 10.1523/jneurosci.184707.2007

Kann, O., Papageorgiou, I. E., and Draguhn, A. (2014). Highly energized inhibitory interneurons are a central element for information processing in cortical networks. J. Cereb. Blood Flow Metab. 34, 1270-1282. doi: 10.1038/jcbfm.20 14.104

Krasnow, A. M., and Attwell, D. (2016). NMDA receptors: power switches for oligodendrocytes. Neuron 91, 3-5. doi: 10.1016/j.neuron.2016. 06.023

Lai, H. C., and Jan, L. Y. (2006). The distribution and targeting of neuronal voltagegated ion channels. Nat. Rev. Neurosci. 7, 548-562. doi: 10.1038/nrn1938

Manganas, L. N., and Trimmer, J. S. (2000). Subunit composition determines Kv1 potassium channel surface expression. J. Biol. Chem. 275, 29685-29693. doi: $10.1074 /$ jbc.m005010200

Melzer, S., Michael, M., Caputi, A., Eliava, M., Fuchs, E. C., Whittington, M. A., et al. (2012). Long-range-projecting GABAergic neurons modulate inhibition in hippocampus and entorhinal cortex. Science 335, 1506-1510. doi: 10.1126/ science. 1217139

Micheva, K. D., Wolman, D., Mensh, B. D., Pax, E., Buchanan, J., Smith, S. J., et al. (2016). A large fraction of neocortical myelin ensheathes axons of local inhibitory neurons. Elife 5:e15784.

Ogawa, Y., Horresh, I., Trimmer, J. S., Bredt, D. S., Peles, E., and Rasband, M. N. (2008). Postsynaptic density-93 clusters Kv1 channels at axon initial segments independently of Caspr2. J. Neurosci. 28, 5731-5739. doi: 10.1523/JNEUROSCI. 4431-07.2008

Ogawa, Y., Schafer, D. P., Horresh, I., Bar, V., Hales, K., Yang, Y., et al. (2006). Spectrins and ankyrinB constitute a specialized paranodal cytoskeleton. J. Neurosci. 26, 5230-5239. doi: 10.1523/jneurosci.0425-06.2006

Pinatel, D., Hivert, B., Boucraut, J., Saint-Martin, M., Rogemond, V., Zoupi, L., et al. (2015). Inhibitory axons are targeted in hippocampal cell culture by antiCaspr2 autoantibodies associated with limbic encephalitis. Front. Cell Neurosci. 9:265. doi: $10.3389 /$ fncel.2015.00265

Pinatel, D., Hivert, B., Saint-Martin, M., Noraz, N., Savvaki, M., Karagogeos, D., et al. (2017). The Kv1-associated molecules TAG-1 and Caspr2 are selectively targeted to the axon initial segment in hippocampal neurons. J. Cell Sci. 130, 2209-2220. doi: 10.1242/jcs.202267

Poliak, S., Gollan, L., Salomon, D., Berglund, E. O., Ohara, R., Ranscht, B., et al. (2001). Localization of Caspr2 in myelinated nerves depends on axon-glia interactions and the generation of barriers along the axon. J. Neurosci. 21, 7568-7575. doi: 10.1523/jneurosci.21-19-07568.2001

Poliak, S., Salomon, D., Elhanany, H., Sabanay, H., Kiernan, B., Pevny, L., et al. (2003). Juxtaparanodal clustering of Shaker-like $\mathrm{K}+$ channels in myelinated axons depends on Caspr2 and TAG-1. J. Cell Biol. 162, 1149-1160. doi: 10. 1083/jcb.200305018

Rasband, M. N. (1998). Clustered K+ channel complexes in axons. Neurosci. Lett. 486, 101-106. doi: 10.1016/j.neulet.2010.08.081

Salzer, J. L. (2008). Switching myelination on and off. J. Cell Biol. 181, 575-577. doi: $10.1083 /$ jcb. 200804136

Sanchez-Ponce, D., Defelipe, J., Garrido, J. J., and Munoz, A. (2012). Developmental expression of Kv potassium channels at the axon initial segment of cultured hippocampal neurons. PLoS One 7:e48557. doi: 10.1371/journal. pone. 0048557

Savvaki, M., Panagiotaropoulos, T., Stamatakis, A., Sargiannidou, I., Karatzioula, P., Watanabe, K., et al. (2008). Impairment of learning and memory in TAG-1 deficient mice associated with shorter CNS internodes and disrupted juxtaparanodes. Mol. Cell. Neurosci. 39, 478-490. doi: 10.1016/j.mcn.2008. 07.025

Savvaki, M., Theodorakis, K., Zoupi, L., Stamatakis, A., Tivodar, S., Kyriacou, K., et al. (2010). The expression of TAG-1 in glial cells is sufficient for the formation of the juxtaparanodal complex and the phenotypic rescue of tag-1 homozygous mutants in the CNS. J. Neurosci. 30, 13943-13954. doi: 10.1523/JNEUROSCI. 2574- 10.2010

Scott, R., Sanchez-Aguilera, A., Van Elst, K., Lim, L., Dehorter, N., Bae, S. E., et al. (2017). Loss of Cntnap2 causes axonal excitability deficits, developmental delay in cortical myelination, and abnormal stereotyped motor behavior. Cereb. Cortex 29, 586-597. doi: 10.1093/cercor/bhx341 
Sherman, D. L., Tait, S., Melrose, S., Johnson, R., Zonta, B., Court, F. A., et al. (2005). Neurofascins are required to establish axonal domains for saltatory conduction. Neuron 48, 737-742. doi: 10.1016/j.neuron.2005. 10.019

Stedehouder, J., Couey, J. J., Brizee, D., Hosseini, B., Slotman, J. A., Dirven, C. M. F., et al. (2017). Fast-spiking parvalbumin interneurons are frequently myelinated in the cerebral cortex of mice and humans. Cereb. Cortex 27, 5001-5013. doi: $10.1093 /$ cercor/bhx203

Traka, M., Goutebroze, L., Denisenko, N., Bessa, M., Nifli, A., Havaki, S., et al. (2003). Association of TAG-1 with Caspr2 is essential for the molecular organization of juxtaparanodal regions of myelinated fibers. J. Cell. Biol. 162, 1161-1172. doi: $10.1083 /$ jcb. 200305078

Trimmer, J. S. (2015). Subcellular localization of K+ channels in mammalian brain neurons: remarkable precision in the midst of extraordinary complexity. Neuron 85, 238-256. doi: 10.1016/j.neuron.2014.12.042

Tzimourakas, A., Giasemi, S., Mouratidou, M., and Karagogeos, D. (2007). Structure-function analysis of protein complexes involved in the molecular architecture of juxtaparanodal regions of myelinated fibers. Biotechnol. J. 2, 577-583. doi: 10.1002/biot.200700023

Vabnick, I., Trimmer, J. S., Schwarz, T. L., Levinson, S. R., Risal, D., and Shrager, P. (1999). Dynamic potassium channel distributions during axonal development prevent aberrant firing patterns. J. Neurosci. 19, 747-758. doi: 10.1523/ jneurosci.19-02-00747.1999
Zhang, C., Susuki, K., Zollinger, D. R., Dupree, J. L., and Rasband, M. N. (2013) Membrane domain organization of myelinated axons requires betaII spectrin. J. Cell Biol. 203, 437-443. doi: 10.1083/jcb.201308116

Zhang, Y., Bekku, Y., Dzhashiashvili, Y., Armenti, S., Meng, X., Sasaki, Y., et al. (2012). Assembly and maintenance of nodes of ranvier rely on distinct sources of proteins and targeting mechanisms. Neuron 73, 92-107. doi: 10.1016/j. neuron.2011.10.016

Zoupi, L., Savvaki, M., Kalemaki, K., Kalafatakis, I., Sidiropoulou, K., and Karagogeos, D. (2018). The function of contactin-2/TAG-1 in oligodendrocytes in health and demyelinating pathology. Glia 66, 576-591. doi: 10.1002/glia. 23266

Conflict of Interest Statement: The authors declare that the research was conducted in the absence of any commercial or financial relationships that could be construed as a potential conflict of interest.

Copyright (c) 2019 Bonetto, Hivert, Goutebroze, Karagogeos, Crépel and FaivreSarrailh. This is an open-access article distributed under the terms of the Creative Commons Attribution License (CC BY). The use, distribution or reproduction in other forums is permitted, provided the original author(s) and the copyright owner(s) are credited and that the original publication in this journal is cited, in accordance with accepted academic practice. No use, distribution or reproduction is permitted which does not comply with these terms. 